\title{
Regulation of primitive hematopoiesis in zebrafish embryos by the death receptor gene
}

\author{
Tommy T. Kwan ${ }^{\mathrm{a}}$, Raymond Liang ${ }^{\mathrm{a}}$, Catherine M. Verfaillie ${ }^{\mathrm{c}}$, \\ Stephen C. Ekker ${ }^{\mathrm{d}}$, Li C. Chan ${ }^{\mathrm{b}}$, Shuo Lin ${ }^{\mathrm{e}}$, and Anskar Y.H. Leung ${ }^{\mathrm{a}}$ \\ Departments of ${ }^{\mathrm{a}}$ Medicine and ${ }^{\mathrm{b}}$ Pathology, University of Hong Kong, Hong Kong; \\ ${ }^{\mathrm{c}}$ Stem Cell Institute and ${ }^{\mathrm{d} D e p a r t m e n t ~ o f ~ G e n e t i c s, ~ C e l l ~ B i o l o g y ~ a n d ~ D e v e l o p m e n t, ~ U n i v e r s i t y ~ o f ~ M i n n e s o t a, ~}$ \\ Minn., USA; ${ }^{\mathrm{e} D e p a r t m e n t}$ of Molecular, Cell and Developmental Biology, University of California, Los Angeles, Calif., USA
}

(Received 20 July 2005; revised 13 September 2005; accepted 29 September 2005)

\begin{abstract}
Objective. We investigated the regulatory mechanism of primitive hematopoiesis in zebrafish (Danio rerio) embryos with particular reference to the role of a death receptor $(\mathrm{zDR})$ gene, based on a morpholino (MO) knockdown approach.

Methods. MOs targeting the zDR and chordin (Chd) were injected into naturally spawned embryos at one- to four-cell stage. A random sequence (RS) MO was used as a control. Effects on hemoglobin formation (Hb), apoptosis, and lineage-specific gene expression were examined. Embryos injected with $\mathrm{zDR}$, Chd, and RS-MOs were denoted $\mathrm{zDR}^{\mathrm{mo}}$, $\mathrm{zChd}^{\mathrm{mo}}$, and $z_{R S}{ }^{\text {mo }}$, respectively. Those coinjected with Chd+zDR-MOs and Chd+RS-MOs were abbreviated $\mathrm{zChd}^{\mathrm{D}} \mathrm{DR} \mathrm{R}^{\mathrm{mo}}$ and $\mathrm{zChd}+\mathrm{RS}^{\mathrm{mo}}$.

Results. zDR mRNA expression was restricted to the intermediate cell mass of wild-type (WT) and $\mathrm{zChd}^{\mathrm{mo}}$ embryos. At 48 hours postfertilization, $\mathrm{zDR}^{\mathrm{mo}}$ embryos showed increased $\mathrm{Hb}$ compared with WT or $\mathrm{zRS}^{\mathrm{mo}}$ embryos $\left(2.36 \times 10^{-2} \pm 1.13 \times 10^{-3}\right.$ vs $1.85 \times 10^{-2} \pm 5.60$ $\times 10^{-4}$ vs $\left.1.79 \times 10^{-2} \pm 1.31 \times 10^{-3} \mathrm{U}, p<0.05\right)$. $\mathrm{zChd}^{-\mathrm{DC}} \mathrm{R}^{\mathrm{mo}}$ embryos also showed increased Hb compared with $\mathrm{zChd}^{\mathrm{mo}}$ or $\mathrm{zChd}+\mathrm{RS}^{\mathrm{mo}}$ embryos $\left(4.60 \times 10^{-2} \pm 2.79 \times 10^{-3}\right.$ vs $3.17 \times 10^{-2} \pm 1.07 \times 10^{-3}$ vs $\left.3.05 \times 10^{-2} \pm 1.25 \times 10^{-3} \mathrm{U}, p<0.05\right)$. zDR-MO reduced apoptosis, as shown by reduced terminal transferase-mediated dUTP nick end-labeling staining in $\mathrm{zChd}+\mathrm{DR}^{\mathrm{mo}}$ compared with $\mathrm{zChd}+\mathrm{RS}^{\mathrm{mo}}$ embryos and caspase-3 activity in $\mathrm{zDR}^{\mathrm{mo}} \mathrm{vs}$

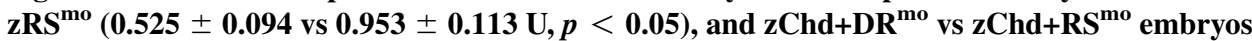
$(0.247 \pm 0.121$ vs $1.180 \pm 0.082, p<0.05) . z^{2}$ Chd+DR ${ }^{\text {mo }}$ embryos showed upregulation of erythroid-specific embryonic hemoglobin gene expression but not that of a myeloid-specific myeloperoxidase gene.

Conclusion. Knockdown of zDR in zebrafish embryos decreased apoptosis and increased $\mathrm{Hb}$, suggesting that zDR may regulate primitive hematopoiesis during development. (C) 2006 International Society for Experimental Hematology. Published by Elsevier Inc.
\end{abstract}

Blood formation during embryonic development in vertebrates is characterized by successive waves of primitive and definitive hematopoiesis. In mammals, primitive and definitive hematopoiesis arises from the extraembryonic yolk sac and the aorto-gonado-mesonephros region, respectively [1]. In zebrafish, primitive hematopoiesis arises from

Offprint requests to: Anskar Y. H. Leung, M.D., Ph.D., Department of Medicine, Professorial Block, Queen Mary Hospital, Pokfulam Road, Hong Kong; E-mail: ayhleung@hku.hk the intermediate cell mass (ICM) [2]. Primitive circulation begins at 24 to 26 hours postfertilization (hpf) and is primarily erythroid in lineage. This primitive wave lasts 3 to 4 days and is followed by a definitive wave that arises at $48 \mathrm{hpf}$ from the ventral wall of the dorsal aorta [2]. The distinctive nature of these waves is exemplified by the bloodless mutants that have defective primitive but normal definitive hematopoiesis [3].

Data from the studies of Xenopus [4,5], zebrafish [6], and mice [7] showed that primitive hematopoiesis is specified by bone morphogenetic proteins (BMPs). In zebrafish, 
both chemical-induced mutagenesis and gene knockdown studies have identified factors that are important for the initiation of primitive hematopoiesis [2]. However, the regulatory mechanism whereby primitive hematopoiesis naturally regresses as it is taken over by the definitive wave has not been elucidated.

In vitro studies using human hematopoietic progenitor cells have demonstrated negative regulation of erythropoiesis by apoptotic signals mediated by multiple death receptors, including Fas, tumor necrosis factor (TNF) receptor 1, TNF-related apoptosis-inducing ligand (TRAIL) receptor-1 and -2 [8]. In zebrafish, the gene encoding for a death receptor (zDR) has been cloned that represents a novel member of the TNF receptor family [9]. It comprises two conserved extracellular cysteine-rich domains, a transmembrane domain, and an intracellular death domain that is similar to those of previously described TNF receptor family. zDR is expressed exclusively in hematopoietic tissues, including embryonic ICM, adult kidneys, and circulating erythroid cells. Adult zebrafish that carry a dominant negative form of zDR exhibit features of polycythemia, suggesting that $\mathrm{zDR}$ is important for regulation of erythropoiesis in definitive hematopoiesis [9]. However, it is not clear if it regulates primitive hematopoiesis during early embryonic development.

We have previously shown that knockdown of chordin (Chd) gene [10] (a BMP antagonist) using morpholinos (MOs) results in expanded hematopoiesis and concomitantly increased apoptosis in the ICM of zebrafish embryos [11]. Gene array analysis identified upregulation of the zDR gene, among others, in the tail of these $\left(\mathrm{zChd}^{\mathrm{MO}}\right) \mathrm{em}-$ bryos. In this study, we examine the role of zDR and apoptosis in the regulation of primitive hematopoiesis in zebrafish embryos. We also investigate whether increased expression of zDR may be, at least in part, responsible for apoptosis of hematopoietic cells in $\mathrm{zChd}^{\mathrm{MO}}$ embryos.

\section{Materials and methods}

\section{Zebrafish}

Danio rerio (wild-type) were obtained from a local aquarium and maintained and raised under standard conditions at $28^{\circ} \mathrm{C}$. Wildtype embryos were obtained from natural spawning and were staged according to Kimmel et al. [12].

\section{Morpholinos injection}

Antisense MOs (Gene Tools, LLC, Philomath, OR, USA) were designed to target at the $5^{\prime}$ untranslated region or flanking, including the initiation codon of the respective genes. MO sequences were: Chd-MO: 5' -ATCCAC AGCAGCCCCTCCATCATCC-3'; zDR-MO 5'-GTAATGTATCTCATAGTAGTCCAAT-3'. Random sequence (RS) MO as control for zDR-MO: 5' -CCTCTTACCT CAGTTACAATTTATA- ${ }^{\prime}$. Search of the TIGR database did not identify sequence similarity with any known zebrafish gene with the RS-MO. Solutions were prepared and injected at one- to four-cell stage as described [13]. Injected embryos were maintained at $28^{\circ} \mathrm{C}$ until analyzed.
Whole-mount in situ hybridization

Identification of zebrafish hematopoietic death receptor (ZH-DR) and synthesis of riboprobes for ZH-DR have been described previously [9]. Whole mount in situ hybridization was performed according to standard protocols. Digoxigenin-labeled probes were detected with alkaline phosphatase conjugated with anti-digoxigenin antibody. 5-Bromo-4-chloro-3-indolyl phosphate/nitro blue tetrazolium was used as substrate to develop purple color (Roche Molecular Biochemicals, Mannheim, Germany).

\section{Dianisidine staining}

Embryos were dechorionated at $48 \mathrm{hpf}$ and stained for 15 minutes in the dark in O-dianisidine $(0.6 \mathrm{mg} / \mathrm{mL})$, sodium acetate $(0.01 \mathrm{M}$, $\mathrm{pH} 4.5), \mathrm{H}_{2} \mathrm{O}_{2}(0.65 \%)$, and ethanol (40\%) (Sigma, St Louis, MO, USA). Presence of brown coloration indicates presence of hemoglobin in zebrafish embryos.

\section{Hemoglobin quantification}

Hemoglobin $(\mathrm{Hb})$ content in zebrafish embryos was quantified by a modified cyanomethemoglobin method as per manufacturer's protocol. Embryos at 24 and $48 \mathrm{hpf}$ were dechorionated and sonicated in Drabkin solution (Sigma) and centrifuged at 14,000 rpm for 15 minutes. Absorbance of the supernatant, measured spectrometrically at $540 \mathrm{~nm}$, has been shown to correlate with the amount of $\mathrm{Hb}$ in adult fish blood in a linear fashion [9].

\section{TUNEL assay}

Apoptosis in the ICM of zebrafish embryos was examined using terminal transferase-mediated dUTP nick end-labeling (TUNEL) assay as per manufacturer's protocol with some modifications (ApopTag Fluoresecent direct In situ Apoptosis Detection Kit; Chemicon, Temecula, CA, USA). Briefly, dechorionated and staged embryos were fixed in $4 \%$ paraformaldehyde and stored in methanol at $-20^{\circ} \mathrm{C}$. They were rehydrated, permeabilized by proteinase $\mathrm{K}$ (Sigma) $(10 \mu \mathrm{g} / \mathrm{mL})$ and refixed in paraformaldehyde. After washing, embryos were incubated in equilibration buffer for 1 hour, followed by overnight incubation with terminal deoxynucleotidyl transferase and digoxigenin-labeled dUTP. The labeling reaction was stopped by the stop/wash buffer. Embryos were incubated with fluorescein-tagged anti-Dig antibody, washed, and thereafter analyzed under a fluorescence microscope. TUNEL assay was also performed similarly in paraffin sections (5 $\mu \mathrm{m})$ of zebrafish embryos. Serial sectioning was performed to compare the fluorescent signal against a hematoxylin and eosin histological section.

\section{Caspase-3 assay}

Caspase- 3 activity in zebrafish embryos was examined using BD ApoAlert Caspase-3 Assay as per manufacturer's protocol (BD ApoAlert Caspase-3 Colorimetric Assay Kit; BD Biosciences Clontech, Mountain View, CA, USA). Groups of 30 embryos at $48 \mathrm{hpf}$ were used. A total of $50 \mu \mathrm{L}$ chilled cell lysis buffer was added to each sample, and the sample was then homogenized and incubated on ice for 10 minutes. The sample was vortexed and centrifuged at $14,000 \mathrm{rpm}$ for 10 minutes in a $4^{\circ} \mathrm{C}$ refrigerated microfuge. Supernatant was then transferred to a new microcentrifuge tube and placed on ice. Fifty microliters $2 \times$ reaction buffer/ dithiothreitol mix was added to the supernatant. To verify that the signal detected was due to protease activity, $1 \mu \mathrm{L}$ of caspase- 3 inhibitor (DEVD-fmk) was added in negative control samples and $1 \mu \mathrm{L}$ dimethylsulfoxide was added to samples without inhibitor 
to ensure all samples were tested under similar conditions. Five microliters $1 \mathrm{mM}$ caspase-3 substrate (DEVE-pNA; $50 \mathrm{uM}$ final concentration) was then added to each tube and all tubes were incubated at $37^{\circ} \mathrm{C}$ for 1 hour in a water bath. Samples were then read at $405 \mathrm{~nm}$ in a spectrophotometer using a $100-\mu \mathrm{L}$ quartz cuvette.

\section{Quantitative polymerase chain reaction assay}

Real-time quantitative polymerase chain reaction (Q-PCR) was performed to examine relative gene expression of erythroid ( $\alpha$ and $\beta$ embryonic $\mathrm{Hb}$, $\alpha \mathrm{he} 1$ and $\beta$ he 1 ) and myeloid lineages (myeloperoxidase [mpo]) using the ABI Prism 7700 Sequence Detector (Applied Biosystems, Foster City, CA, USA). Embryos at $48 \mathrm{hpf}$ were dechorionated and RNA was extracted using Trizol reagent as per manufacturer's protocol (Invitrogen, Carlsbad, CA, USA). Thirty embryos were included in each experiment. RNA was reverse transcribed and PCR was performed using the SYBR green method. Sequences of primers used for Q-PCR were as follows:

ahe $1_{\mathrm{f}}: 5^{\prime}$ AAG ATT CTG TCC CAC AAC ATC C 3' ahe $1_{\mathrm{r}}: 5^{\prime}$ GCT GAG AGG AAC TTG TCC ATT G 3' Bhe $1_{\mathrm{f}}: 5^{\prime}$ GTC TTA TCG TGT ACC CCT GGA C 3' Bhe $1_{\mathrm{r}}: 5^{\prime}$ GAC CCT TGA GCA CAG TTT TAC C 3' mpo: 5' $^{\prime}$ TGC CTA CAT GTT TGG TGA AGT C 3' mpor: 5' CAT TAG TAA GGT CCC GCA GTT C 3'

Before the start of the project, a group of 40 embryos at $48 \mathrm{hpf}$ were dechorionated and RNA was extracted and reverse transcribed. Serial dilutions of cDNA from that sample corresponded to $25,2.5,0.25,0.025,0.0025 \mathrm{ng}$ (for $\alpha$ he 1 and $\beta$ he1 Q-PCR) and $125 \mathrm{ng}, 25,5,1,0.2 \mathrm{ng}$ (for mpo Q-PCR) of input RNA were frozen at $-20^{\circ} \mathrm{C}$ in small aliquots (standards). Once thawed, each aliquot was used only once.

Standard curves for the quantification of $\alpha$ he1, $\beta$ he1, and mpo were constructed by plotting the $\mathrm{C}_{\mathrm{T}} \mathrm{S}$ against the logarithm of the starting amount of serial dilution of standards. Each sample was tested in triplicates, their respective $\mathrm{C}_{\mathrm{T}} \mathrm{S}$ were determined and the initial amount of gene expression calculated from the standard curve. Only groups of embryos at the same developmental stage were compared and results (which corresponded to the input RNA in the standards) were expressed as ratios.

\section{Statistical analysis}

Comparisons of numerical data were evaluated by Mann-Whitney $U$-test and Kruskal-Wallis test. A $p$-value of $<0.05$ was considered statistically significant.

\section{Results}

Expression of $z D R$ in wild-type and $z C h d^{m o}$ embryos zDR mRNA expression is first detected in the ventral posterior region of zebrafish embryos at 10-somite stage. Between the 15- and 20-somite stages, its expression is restricted to the ICM [9]. We compared the late zDR expression in wild-type (WT) and $\mathrm{zChd}^{\mathrm{mo}}$ embryos at 24 and 48 hpf to investigate if it is upregulated in the latter embryos in which primitive hematopoiesis is accentuated (Fig. 1a-d). At $24 \mathrm{hpf}, \mathrm{zDR}$ is expressed in the ICM of WT embryos. In $\mathrm{zChd}^{\mathrm{mo}}$ embryos, zDR is upregulated in the expanded posterior ICM. At $48 \mathrm{hpf}$, zDR expression is virtually undetectable in WT and faintly expressed in the ICM of $\mathrm{zChd}^{\mathrm{mo}}$ embryos.

\section{Effects of zDR-MO on zebrafish morphology}

Adult zebrafish carrying a dominant negative form of zDR exhibits increased $\mathrm{Hb}$ content reminiscent of human polycythemia [9]. This has led us to examine the effect of zDR-MO in zebrafish embryos. zDR-MOs were injected at $3 \mathrm{ng}$ in all experiments because initial dose-finding studies showed toxicity and mortality at higher doses (not shown). There was no difference in gross morphology between $\mathrm{zDR}^{\mathrm{mo}}$ and WT embryos (Fig. 1e and $\mathrm{f}$ ) and between $\mathrm{zChd}+\mathrm{DR}^{\mathrm{mo}}$ and $\mathrm{zChd}{ }^{\mathrm{mo}}$ embryos (Fig. $1 \mathrm{~g}$ and $\mathrm{h}$ ) at either 48 hpf or 24 hpf (not shown).

\section{Effects of zDR-MO on embryo Hb content}

We next examined the effects of zDR-MO on primitive hematopoiesis, which can be assessed at 48 hpf by the presence of $\mathrm{Hb}$ stainable by O-dianisidine. In WT embryos, $\mathrm{Hb}$ staining was positive in the great vein on the surface of the yolk (Duct of Cuvier [DC]) (Fig. 1i and j). In $\mathrm{zDR}^{\mathrm{mo}}$ embryos, the axial circulation and the DC appeared more congested (inserts). In $\mathrm{zChd}^{\mathrm{mo}}$ embryos, $\mathrm{Hb}$ staining is significantly increased in the ICM as well as the DC, which is further enhanced in $\mathrm{zChd}+\mathrm{DR}^{\mathrm{mo}}$ embryos (Fig. 1k and 1). This was confirmed with a quantitative assay using a modified cyanomethemoglobin method in which the absorbance varies linearly with the amount of $\mathrm{Hb}$ [9]. In our initial validation of this method, published by Long et al. [9], we established that the absorbance also varied linearly with the number of input $48 \mathrm{hpf}$ embryos over the range of 5 to 40 embryos (data not shown). Therefore, in all subsequent experiments, the number of input embryos was standardized to 20 per experiment so that comparison of $\mathrm{Hb}$ contents between different experimental groups can be made. As a control, embryos were injected with an equivalent amount of RS-MO. At $24 \mathrm{hpf}$, the $\mathrm{Hb}$ content is low and there is no significant difference between $\mathrm{WT}, \mathrm{zDR}^{\mathrm{mo}}$, $\mathrm{zChd}^{\mathrm{mo}}$, and zChd $+\mathrm{DR}^{\mathrm{mo}}$ embryos (Fig. 2). At $48 \mathrm{hpf}$, $\mathrm{zDR}^{\mathrm{mo}}$ embryos showed an increase in $\mathrm{Hb}$ content compared with WT and $\mathrm{zRS}^{\mathrm{mo}}$ embryos $\left(2.36 \times 10^{-2} \pm 1.13\right.$ $\times 10^{-3}$ vs $1.85 \times 10^{-2} \pm 5.60 \times 10^{-4}$ vs $1.79 \times 10^{-2}$ $\left.\pm 1.31 \times 10^{-3} \mathrm{U}, \mathrm{p}<0.05\right)$. $\mathrm{zChd}^{\mathrm{mo}}$ embryos also showed a higher level of $\mathrm{Hb}$ compared with WT and $\mathrm{zRS}^{\mathrm{mo}}$ embryos $\left(3.17 \times 10^{-2} \pm 1.07 \times 10^{-3}\right.$ vs $1.85 \times 10^{-2} \pm 5.60 \times$ $10^{-4}$ vs $\left.1.79 \times 10^{-2} \pm 1.31 \times 10^{-3} \mathrm{U}, \mathrm{p}<0.001\right)$. Coinjection with $\mathrm{zDR}-\mathrm{MO}\left(\mathrm{zChd}+\mathrm{DR}^{\mathrm{mo}}\right.$ embryos) further increased $\mathrm{Hb}$ content $\left(4.60 \times 10^{-2} \pm 2.79 \times 10^{-3}\right.$ vs $3.17 \times$ $\left.10^{-2} \pm 1.07 \times 10^{-3} \mathrm{U}, \mathrm{p}<0.05\right)$ whereas coinjection of zRS-MO (zChd $+\mathrm{RS}^{\text {mo }}$ embryos) $\left(3.05 \times 10^{-2} \pm 1.25\right.$ $\left.\times 10^{-3} \mathrm{U}\right)$ had no additional effect on $\mathrm{Hb}$ content of zChd $^{\text {mo }}$ embryos. 

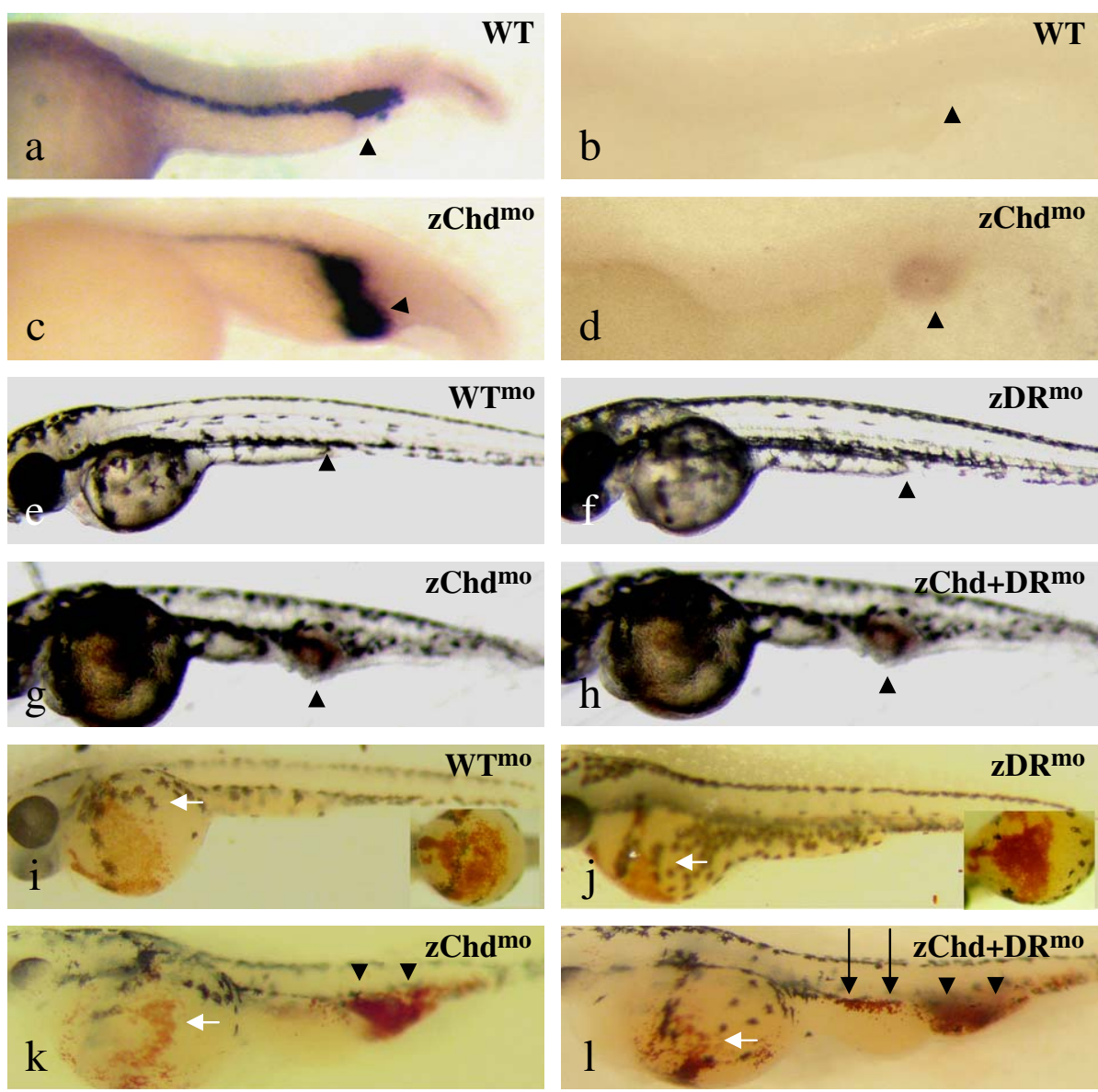

Figure 1. zDR gene expression and the effects of zDR morpholino on primitive hematopoiesis in wild-type (WT) and embryos. (a-d): zDR expression in WT (a,b) and $\mathrm{zCh}^{\text {mo }}$ embryos (c,d) at 24 (a,c) and 48 (b,d) hours postfertilization (hpf). (e-h) Gross morphology of 48 hpf WT (e), zDR ${ }^{\text {mo }}$ (f), zChd ${ }^{\text {mo }}$ (g) and $z$ Chd $+\mathrm{DR}^{\mathrm{mo}}$ (h) embryos. (i-l): O-dianisidine staining at 48 hpf of WT (i), zDR ${ }^{\text {mo }}$ (j), zChd ${ }^{\text {mo }}$ (k), and zChd $+\mathrm{DR}^{\text {mo }}(\mathbf{l})$ embryos. Hemoglobin-containing cells are stained brown in this preparation. Note that circulation of zDR ${ }^{\text {mo }}$ embryos appeared more congested than WT embryos as shown by closeups of the duct of Cuvier (inserts). Similarly, circulation of zChd+DR ${ }^{\text {mo }}$ embryos was more congested than that of zChd ${ }^{\text {mo }}$ embryos. White arrows: Circulation in the duct of Curvier. Black arrowheads: Expanded intermediate cell mass in $z$ Chd ${ }^{\text {mo }}$ embryos with or without coinjection with zDR-MO. Each picture represents typical results out of three experiments (more than 10 embryos in each experiment). Black arrows: More congested axial circulation in embryos injected with zDR-MO.

\section{Effects of zDR-MO on ICM histology}

To have a closer look at the ICM at the cellular level, we examined serial sections of the embryos at 24 and $48 \mathrm{hpf}$. In WT embryos, the ICM contains only hematopoietic cells one- to two-cell layers thick [11]. Hence, comparison between zDR-MO and RS control is difficult. Therefore, we investigated the effects of zDR-MO in the background of ICM expansion in $\mathrm{zChd}^{\mathrm{mo}}$ embryos. At $24 \mathrm{hpf}$, there was no significant difference between $\mathrm{zChd}+\mathrm{DR}^{\mathrm{mo}}$ and $\mathrm{zChd}+\mathrm{RS}^{\mathrm{mo}}$ embryos. At $48 \mathrm{hpf}$, there was a decrease in hematopoietic cell density in the expanded ICM, resulting in cavitations of ICM and the presence of apoptotic bodies in most $z C h d+\mathrm{RS}^{\mathrm{mo}}$ embryos (Fig. 3). In $z \mathrm{Chd}+\mathrm{DR}^{\mathrm{mo}}$ embryos, however, cellular density is much better preserved in most cases, suggesting that knockdown of zDR overcomes the apoptosis seen in ICM of Chd ${ }^{\mathrm{mo}}$ embryos.
Effects of zDR-MO on apoptosis during zebrafish embryonic hematopoiesis

To substantiate that, the increase in $\mathrm{Hb}$ content of the $\mathrm{zDR}^{\mathrm{mo}}$ and $\mathrm{zChd}+\mathrm{DR}^{\mathrm{mo}}$ embryos is related to perturbation of apoptosis, we used the TUNEL assay. In WT and zDR ${ }^{\text {mo }}$ embryos, there was very little apoptotic signal in the ICM area at both 24 (data not shown) and 48 hpf (Fig. 4). In $\mathrm{zChd}^{\mathrm{mo}}$ embryos, apoptotic signals were increased, mostly in the posterior part of the body. At $24 \mathrm{hpf}$, apoptosis occurred predominantly on the surface of the embryos and zDR-MO had no effect on this (data not shown). At 48 hpf, apoptosis was extensive in the expanded ICM of zChd $^{\text {mo }}$ embryos. In zChd $+\mathrm{DR}^{\text {mo }}$ embryos, however, apoptotic signals are significantly reduced, further suggesting that the increased levels of zDR in $\mathrm{zChd}^{\mathrm{mo}}$ embryos may be responsible for the increased apoptosis in this morphant. 


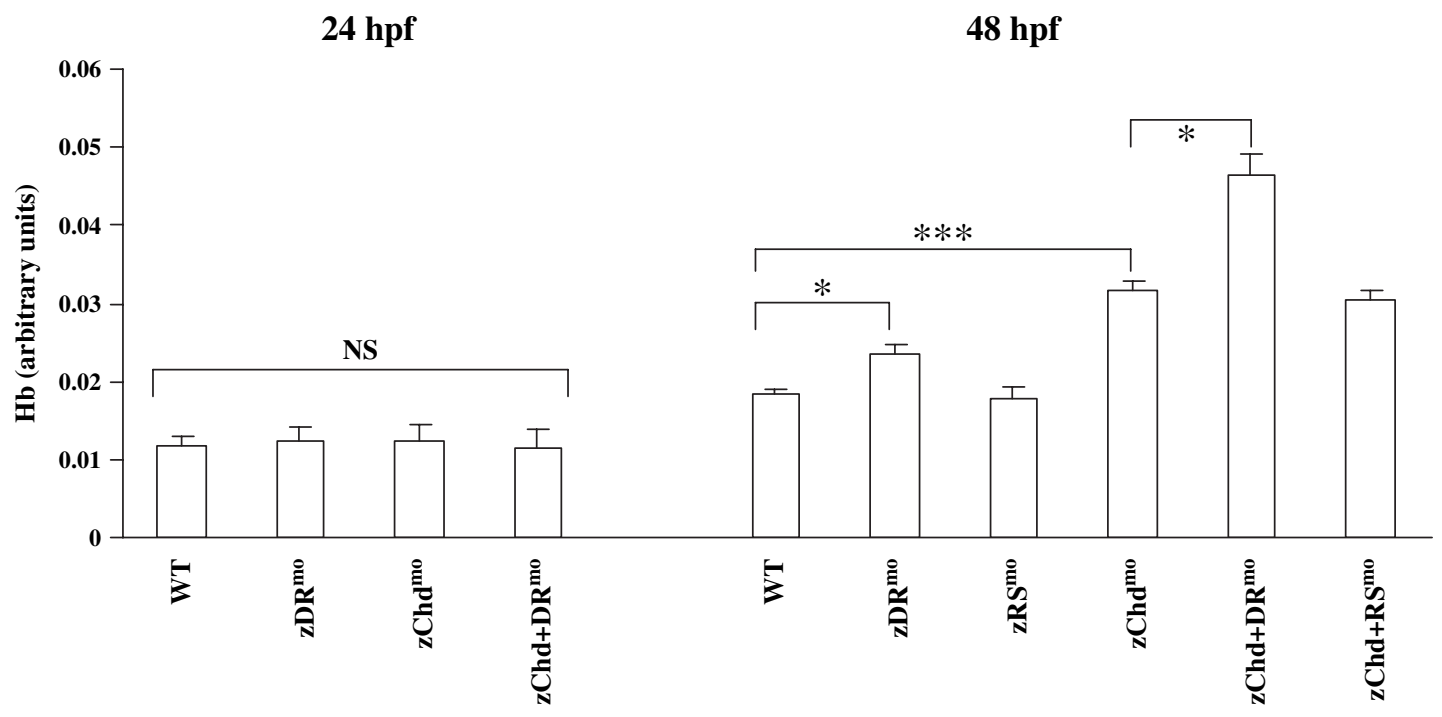

Figure 2. Quantitative effects of zDR-MO on hemoglobin contents in wild-type (WT) and zChd ${ }^{\mathrm{mo}}$ embryos at 24 and 48 hours postfertilization (hpf). Randsom sequence (RS) morpholino (MO) at 3 ng was injected as a control to zDR-MO. * $p<0.05$; *** $p<0.001$ (Mann-Whitney $U$-test and Kruskal-Wallis test). NS $=$ not significant. Results represent mean \pm SEM of three to five separate experiments. Absorbance varies linearly with the number of embryos used in the assay and for standardization 20 embryos were used in each experiment and the groups of embryos for comparison were at similar developmental stage.
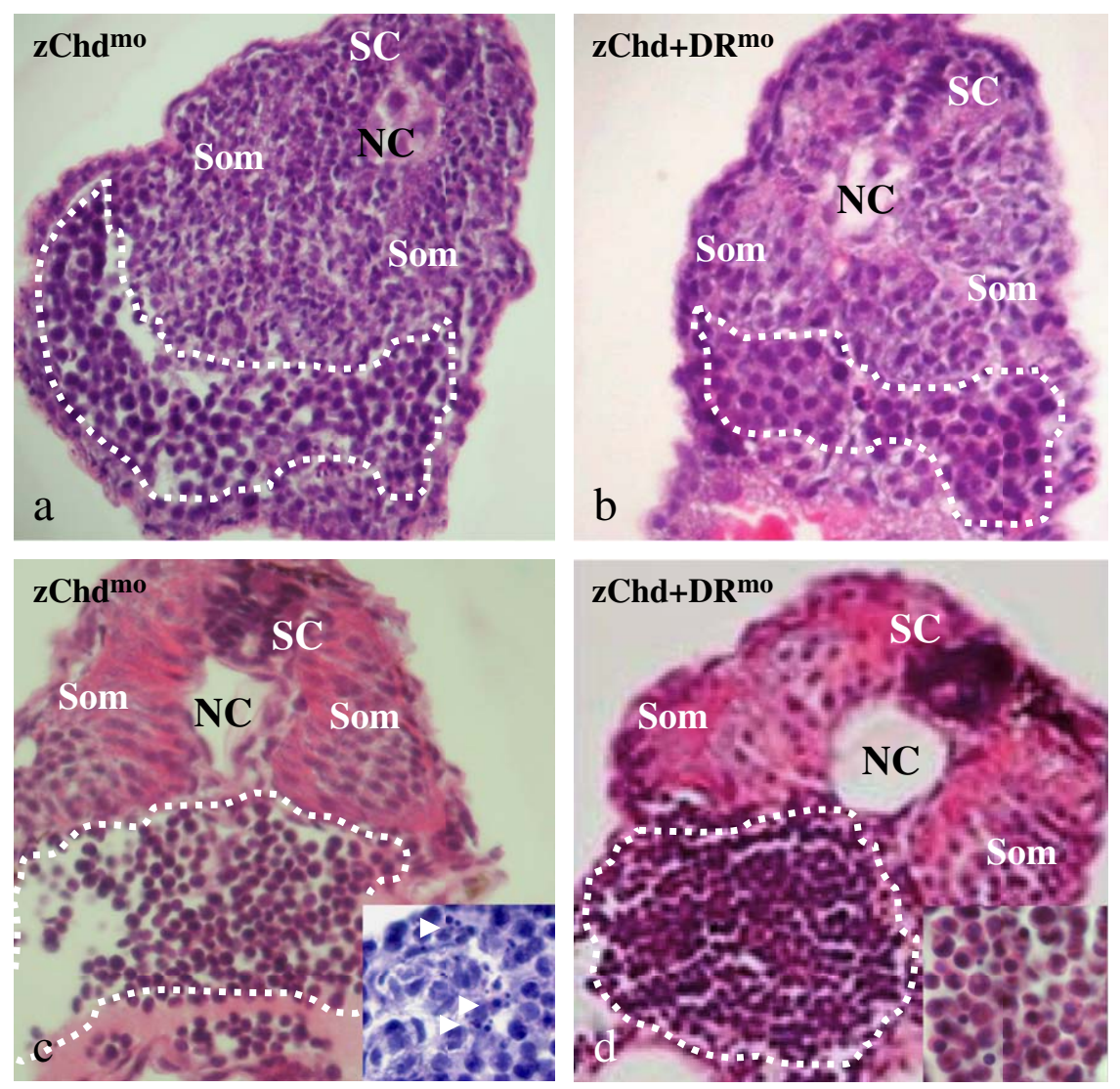

Figure 3. Effects of zDR-MO on the histology of expanded intermediate cell mass (ICM) in $\mathrm{zChd}^{\mathrm{mo}}$ embryos. (a-b): $\mathrm{zChd}^{\mathrm{mo}}(\mathbf{a})$ and $\mathrm{zChd}+\mathrm{DR}{ }^{\mathrm{MO}}(\mathbf{b})$ embryos at 24 hours postfertilization (hpf). (c-d): Corresponding embryos at $48 \mathrm{hpf}$. The insert at (c) showed the frequent occurrence of apoptotic bodies (arrowheads) in $\mathrm{zChd}^{\mathrm{mo}}$ as compared to $\mathrm{zChd}+\mathrm{DR}^{\mathrm{MO}}$ [insert at (d)] embryos at $48 \mathrm{hpf}$. All pictures are coronal sections stained with hematoxylin and eosin. $\mathrm{NC}=$ notochord; $\mathrm{SC}=$ spinal cord; Som $=$ somites. The expanded ICM was outlined by dotted white lines in each figure. 

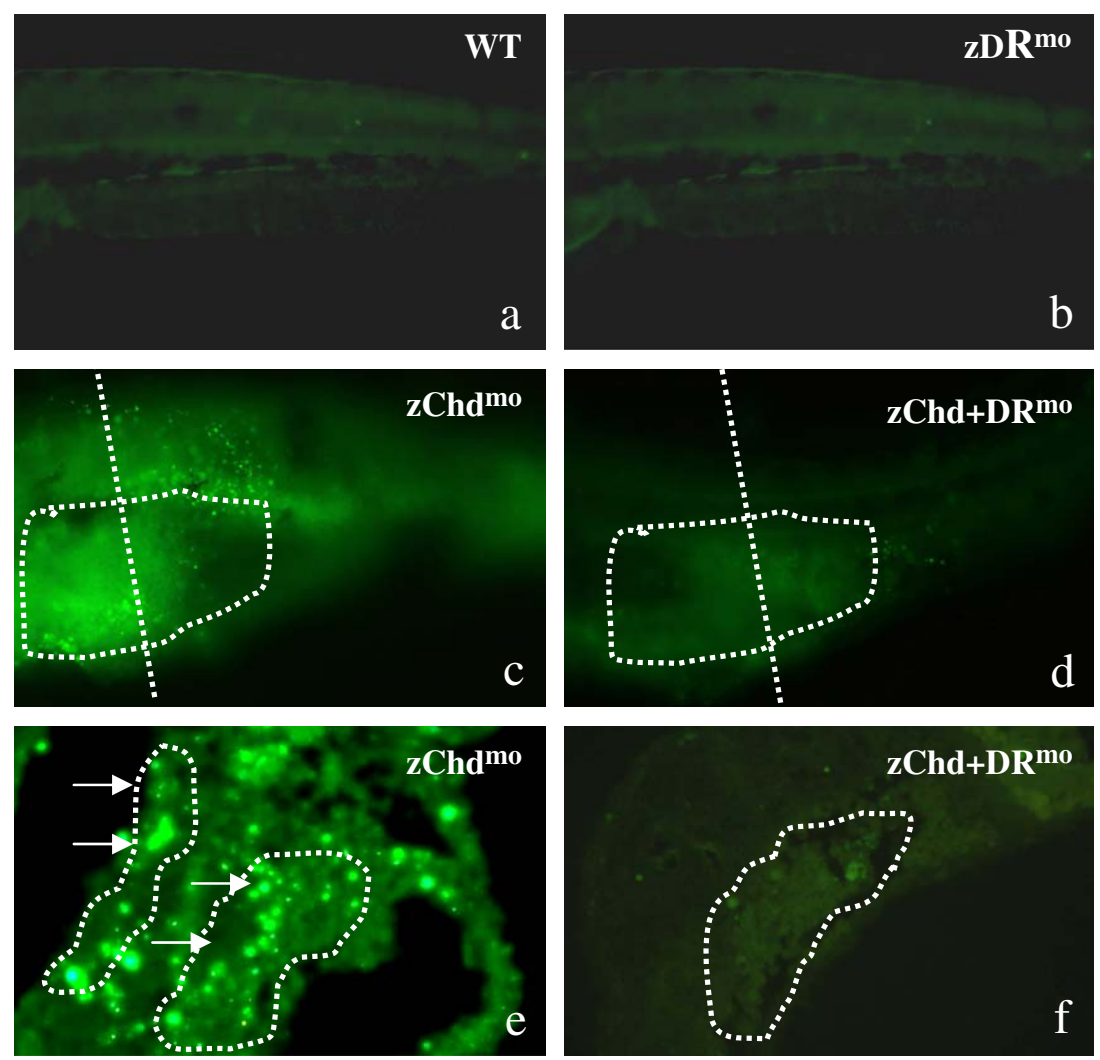

Figure 4. Effects of zDR and chordin-morpholino (MO) injection on apoptosis of intermediate cell mass (ICM) during zebrafish development. (a-b): ICM of wild-type (WT) (a) and zDR ${ }^{\mathrm{MO}}$ embryos (b) showing little fluorescence by the TUNEL assay. (c,d): ICM of zChd ${ }^{\text {mo }}$ embryos at 48 hours postfertilization (hpf) showing terminal transferase mediated dUTP nick end-labeling (TUNEL) staining (apoptosis) in the ICM and adjacent tissues (c). Reduced fluorescence at ICM in zChd $+\mathrm{DR}^{\mathrm{MO}}$ embryos (d). (e,f): TUNEL staining on sections of ICM from zChd ${ }^{\mathrm{mo}}$ (e) and zChd $+\mathrm{DR}^{\mathrm{MO}}$ (f) embryos. Embryos were staged at 48 hpf and each picture represent typical results of at least three experiments. Dotted lines defined the hematopoietic compartment of expanded ICM as inferred from adjacent hematoxylin and eosin sections. Oblique lines indicate the estimated locations of sections as shown in (e) and (f). Injection random sequence $\mathrm{MO}$ at $3 \mathrm{ng}$ did not induce changes in TUNEL staining in either WT or zDR ${ }^{\mathrm{MO}}$ embryos (not shown).

\section{Effects of zDR-MO on caspase-3 activity}

We further examined if the reduction of apoptotic signals in $\mathrm{zDR}^{\mathrm{mo}}$ could also be manifested as a reduction of caspase- 3 activity, an effecter caspase for apoptosis. At $48 \mathrm{hpf}$, caspase- 3 activity was significantly reduced in $\mathrm{zDR}^{\mathrm{mo}}$ compared with $\mathrm{zRS}^{\mathrm{mo}}(0.525 \pm 0.094$ vs $0.953 \pm 0.113 \mathrm{U}, p$ $<0.05$ ), and the difference was more prominent between $\mathrm{zChd}+\mathrm{DR}^{\mathrm{mo}}$ and $\mathrm{zChd}+\mathrm{RS}^{\mathrm{mo}}$ embryos $(0.247 \pm 0.121$ vs $1.180 \pm 0.082, p<0.05$ ) (Fig. 5A).

\section{Effects of zDR-MO on hematopoietic gene expression}

Previous studies showed that expression of zDR was restricted to the erythroid lineage of zebrafish embryos [9]. We therefore investigated the effects of zDR gene knockdown on expression of genes encoding for both erythroid ( $\alpha$ and $\beta$ embryonic $\mathrm{Hb}$, $\alpha$ he 1 and $\beta$ he 1 ) as well as myeloid lineages (myeloperoxidase, mpo). At $48 \mathrm{hpf}, \mathrm{zDR}^{\mathrm{mo}}$ showed no difference in ahe1, $\beta$ h1e, or mpo mRNA expression compared with zRS ${ }^{\mathrm{mo}}$ (Fig. 5B). However, in $\mathrm{zChd}^{\mathrm{mo}} \mathrm{em}-$ bryos, zDR-MO (zChd $+\mathrm{DR}^{\mathrm{mo}}$ embryos) increased overall expression of ahe1 (1.6- \pm 0.11 -fold) and $\beta$ he1 (2.2- \pm 0.51 -fold), but not of mpo (0.70- \pm 0.35 -fold) mRNA expression, as compared with zRS-MO (zChd $+\mathrm{RS}^{\mathrm{mo}} \mathrm{em}-$ bryos). These findings further supported the notion that zDR knockdown increased overall erythroid population in the chordin morphant background.

\section{Discussion}

A number of zebrafish genes with homology to mammalian apoptosis regulators have been identified [14]. Recently, transgenic fish that overexpress Bcl-2 [15] and caspase-3 [16] have been generated, showing that these regulators play an important role in the anti- and proapoptotic signaling in zebrafish, similar to their mammalian counterparts. In the present study, knockdown of zDR using MO increased $\mathrm{Hb}$ content in zebrafish embryos, as shown qualitatively by increased density of o-dianisidine staining and quantitatively by a modified cyanomethemoglobin assay. Effects of zDR-MO were specific, as RS-MO has no effect on either $\mathrm{Hb}$ contents, caspase-3 activity, or erythroid gene expression. Moreover, the observation that zDR-MO did not affect embryo morphology is also consistent with the dominant negative zDR model in which early development 

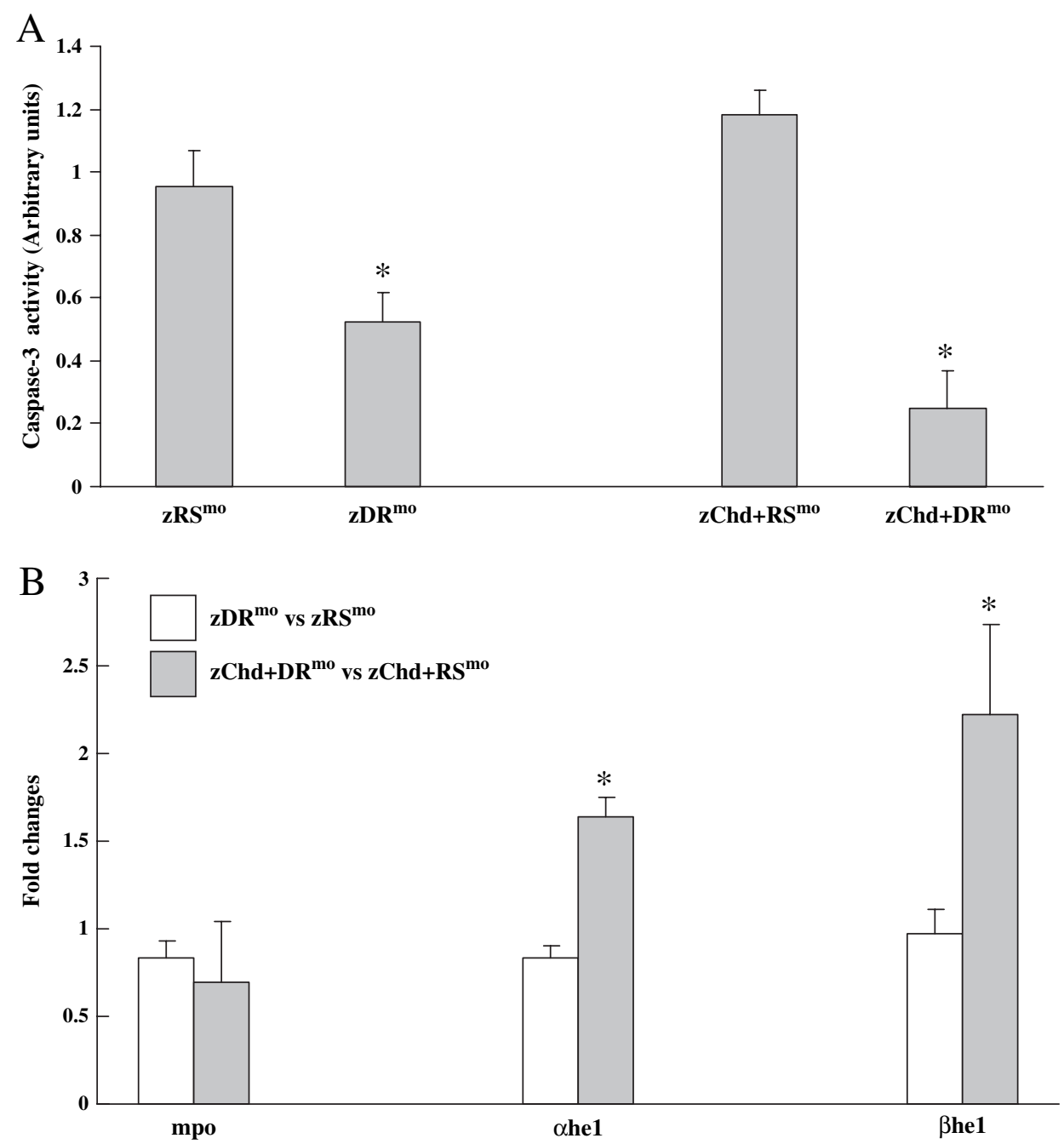

Figure 5. Effects of zDR-MO on caspase-3 activity (A) and gene expression (B) in wild-type (WT) and zChd ${ }^{\mathrm{mo}}$ embryos. Results expressed as mean \pm SEM of three separate experiments. Note that in $(\mathbf{B})$, results represent the fold changes in gene expression of $\mathrm{zDR}^{\mathrm{MO}}$ as compared with random sequence (RS) ${ }^{\mathrm{MO}}$ embryos (empty columns) and of $\mathrm{zChd}+\mathrm{DR}^{\mathrm{MO}}$ as compared with $\mathrm{zChd}+\mathrm{RS}^{\mathrm{MO}}$ (filled columns) embryos. ${ }^{*} p<0.05$.

of embryos was normal, only became polycythemic at adulthood [9]. Effects of zDR-MO were more apparent in the $\mathrm{zChd}+\mathrm{DR}^{\mathrm{mo}}$ embryos that were sensitized to the ventralizing effects of BMP signaling [10,11]. This was accomplished by coinjecting the embryos with chordin morpholino, which can induce an increase in primitive hematopoiesis in a dosedependent and uniform fashion.

The apparent increase in erythropoiesis by zDR-MO can be explained by the diminution of zDR-mediated apoptosis, as shown by the decrease in TUNEL staining and a better preserved hematopoietic cell density in the expanded ICM of the $\mathrm{zChd}+\mathrm{DR}^{\mathrm{mo}}$ embryos as compared with those of the $\mathrm{zChd}+\mathrm{RS}^{\mathrm{mo}}$ embryos. These tests are qualitative and may be subject to observers' bias and variations, especially when the expected effects might be modest. To circumvent these inherent difficulties, we performed, in addition, quantitative assay for caspase- 3 activity, an effector in the apoptosis pathway. Using this assay, we were able to discriminate the difference in baseline apoptosis between the $\mathrm{zDR}^{\mathrm{mo}}$ and $\mathrm{zRS}^{\mathrm{mo}}$ embryos, which was more pronounced in the chordin morphant background. We chose to work on $48 \mathrm{hpf}$, at which apoptosis can be correlated phenotypically with a change in Hb contents. As zDR is expressed exclusively in the hematopoietic tissues (see [9] and Fig. 1), the difference in apoptosis can be explained by the effects of zDR-MO on primitive hematopoiesis. In contrast, very little TUNEL staining were detectable in both $\mathrm{zDR}^{\mathrm{mo}}$ and WT embryos in normal background, suggesting that this assay is not sensitive enough in this circumstances.

Our findings are consistent with previous studies showing that dominant negative zDR induced polycythemia in adult zebrafish [9] and corroborate the notion that receptor-mediated apoptosis plays an important role in the negative regulation of primitive erythropoiesis. However, the downstream components of zDR-mediated apoptosis have not been examined. P53 knockdown in zebrafish embryos 
has been shown to reduce apoptosis induced by drugs and irradiation [17]. On the other hand, overexpression of bcl-2 in zebrafish lymphoid cells was able to protect these tissues against irradiation and steroid-induced apoptosis [15]. Whether they may recapitulate the effects of zDR$\mathrm{MO}$ and hence provide a clue to the downstream pathway for zDR will have to be further examined.

We have also examined the change in gene expression in $\mathrm{zDR}^{\mathrm{mo}}$ and $\mathrm{zChd}+\mathrm{DR}^{\mathrm{mo}}$ embryos. In particular, the overall expression of erythroid-specific genes encoding for $\alpha$ he 1 and $\beta$ he 1 [18] were significantly increased after zDR knockdown in $\mathrm{zChd}^{\mathrm{mo}}$ embryos. The results were consistent with an increase in erythroid cell population in $\mathrm{zChd}+\mathrm{DR}^{\mathrm{mo}}$ as compared with zChd ${ }^{\mathrm{mo}}$ embryos. In contrast, expression of myeloid-associated mpo was not affected. Expression of other hematopoietic genes was not examined and although zDR is normally restricted to erythroid lineage in WT embryos, whether it is similarly restricted in the chordin morphants has not been investigated. The reason for the lack of effects for both erythroid and myeloid genes in WT embryos is also unclear. Notwithstanding these limitations, our results were still consistent with the notion that zDR mediates apoptosis of the primitive erythropoiesis during zebrafish embryonic development and that the increased apoptosis of ICM of $\mathrm{zChd}^{\mathrm{mo}}$ embryos is mediated at least partially by the zDR gene. Previous studies have demonstrated a reciprocal regulatory relationship between erythroid and myeloid cell fate [19] in zebrafish embryos. Such a relationship was not apparent in this study.

Although zDR is likely to be important during primitive hematopoiesis, the increase in $\mathrm{Hb}$ in response to zDR knockdown was only modest. At $48 \mathrm{hpf}$, the $\mathrm{Hb}$ content increased by about $27 \%$ in WT embryos and $45 \%$ in $\mathrm{zChd}^{\mathrm{mo}}$ embryos. This observation thus suggested that other mechanisms may also regulate primitive hematopoiesis during normal development. In quail [20] and murine [5] embryos, apoptosis of primitive hematopoiesis is initiated by withdrawal of retinoid and vascular endothelial growth factor A signaling. Whether these mechanisms are involved in zebrafish would have to be investigated. In addition, the identity of the signaling ligand for zDR has not been elucidated. In vitro, human erythroid progenitor is regulated by various apoptotic signals, including Fas ligand, TNF, and TRAIL [8]. The resulting activation of caspases leads to cleavage of gata-1, an important transcription factor for erythropoiesis. It is unclear whether similar mechanisms might operate in the regulation of primitive hematopoiesis in zebrafish embryos.

In conclusion, the present study showed that knockdown of zDR function in WT and $\mathrm{zChd}^{\mathrm{mo}}$ embryos using MO led to a decrease in apoptosis and an increase in Hb content, suggesting that $\mathrm{zDR}$ may regulate primitive hematopoiesis during zebrafish development. Blastula stage transplantation may help us to define whether the functions of zDR are cell autonomous to the blood lineages.

\section{Acknowledgments}

We thanked Mr. Howard C. H. Chow for performing the reverse transcriptase polymerase chain reaction experiments. Work was supported by RGC grant (HKU 7488/04M).

\section{References}

1. Galloway JL, Zon LI. Ontogeny of hematopoiesis: examining the emergence of hematopoietic cells in the vertebrate embryo. Curr Top Dev Biol. 2003;53:139-158.

2. Davidson AJ, Zon LI. The 'definitive' (and 'primitive') guide to zebrafish hematopoiesis. Oncogene. 2004;23:7233-7246.

3. Liao EC, Trede NS, Ransom D, Zapata A, Kieran M, Zon LI. Non-cell autonomous requirement for the bloodless gene in primitive hematopoiesis of zebrafish. Development. 2002;129:649-659.

4. Maeno M, Mead PE, Kelley C, et al. The role of BMP-4 and GATA-2 in the induction and differentiation of hematopoietic mesoderm in Xenopus laevis. Blood. 1996;88:1965-1972.

5. Schmerer M, Evans T. Primitive erythropoiesis is regulated by Smaddependent signaling in postgastrulation mesoderm. Blood. 2003;102: 3196-3205.

6. Neave B, Holder N, Patient R. A graded response to BMP-4 coordinates patterning of the mesoderm and ectoderm in the zebrafish. Mech Dev. 1997;62:183-195.

7. Baron MH. Embryonic origins of mammalian hematopoiesis. Exp Hematol. 2003;31:1160-1169.

8. De Maria R, Zeuner A, Eramo A, et al. Negative regulation of erythropoiesis by caspase-mediated cleavage of GATA-1. Nature. 1999; 401:489-493.

9. Long Q, Huang H, Shafizadeh E, Liu N, Lin S. Stimulation of erythropoiesis by inhibiting a new hematopoietic death receptor in transgenic zebrafish. Nat Cell Biol. 2000;2:549-552.

10. Hammerschmidt M, Pelegri F, Mullins MC, et al. Dino and mercedes, two genes regulating dorsal development in the zebrafish embryo. Development. 1996;123:95-102.

11. Leung AY, Mendenhall EM, Kwan TT, et al. Characterization of expanded intermediate cell mass in zebrafish chordin morphant embryos. Dev Biol. 2005;277:235-254.

12. Kimmel CB, Ballard WW, Kimmel SR, Ullmann B, Schilling TF. Stages of embryonic development of the zebrafish. Dev Dyn. 1995; 203:253-310.

13. Nasevicius A, Ekker SC. Effective targeted gene 'knockdown' in zebrafish. Nat Genet. 2000;26:216-220.

14. Inohara N, Nunez G. Genes with homology to mammalian apoptosis regulators identified in zebrafish. Cell Death Differ. 2000;7:509-510.

15. Langenau DM, Jette C, Berghmans S, et al. Suppression of apoptosis by bcl-2 overexpression in lymphoid cells of transgenic fish. Blood. 2005;105:3278-3285.

16. Yabu T, Kishi S, Okazaki T, Yamashita M. Characterization of zebrafish caspase-3 and induction of apoptosis through ceramide generation in fish fathead minnow tailbud cells and zebrafish embryos. Biochem J. 2001;360:39-47.

17. Langheinrich U, Hennen E, Stott G, Vacun G. Zebrafish as a model organism for the identification and characterization of drugs and genes affecting p53 signaling. Curr Biol. 2002;12:2023-2028.

18. Brownlie A, Hersey C, Oates AC, et al. Characterization of embryonic globin genes of the zebrafish. Dev Biol. 2003;255:48-61.

19. Galloway JL, Wingert RA, Thisse C, Thisse B, Zon LI. Loss of Gata1 but not Gata2 converts erythropoiesis to myelopoiesis in zebrafish embryos. Dev Cell. 2005;8:109-116.

20. Ghatpande S, Ghatpande A, Sher J, Zile MH, Evans T. Retinoid signaling regulates primitive (yolk sac) hematopoiesis. Blood. 2002; 99:2379-2386. 